COMMENT. The authors advocate a trial of cytochrome C/vitamin B treatment in children with MEM; disease progression is not halted but symptoms may be ameliorated.

\title{
BONE MARROW TRANSPLANT IN ADRENOLEUKODYSTROPHY
}

Three children with adrenoleukodystrophy (ALD) were treated with allogeneic bone marrow transplantation (BMT) at Huddinge University Hospital, Sweden. Patient 1, a boy aged 9, presented with attention deficit disorder and learning disability; MRI showed demyelination in the frontal lobes which progressed over a 4 year follow-up, in association with mental deterioration. The 2 nd and 3rd patients had no symptoms but white matter lesions were present on MRI at diagnosis. One had Addison's disease at 6 years of age, and ALD was confirmed biochemically and by MRI six months later. He showed worsening of attention deficits before, and he died 18 months after BMT. Patient 3 was the younger brother of patient 2, and ALD and Addison's disease were diagnosed at 4 years. This boy is healthy 3.5 years after BMT. (Malm G, Ringden O, Anvret M et al. Treatment of adrenoleukodystrophy with bone marrow transplantation. Acta Paediatr May 1997;86:484-492). (Respond: Dr G Malm, Department of Paediatrics, Huddinge University Hospital, 14186 Huddinge, Sweden).

COMMENT. The authors recommend bone marrow transplantation implemented early in the treatment of ALD, even without symptoms, provided that the MRI shows signs of demyelination and a suitable donor is available.

Attention deficit disorder was the presenting clinical manifestation of ALD in one of these patients, emphasizing the neurologist's role in the management of children with ADHD.

\section{HYPOGLYCEMIA AND COGNITIVE DEFICITS}

Effects of hypoglycemia on cognition were studied using event-related brain-potential (ERP) measures and reaction times at the Otto-von-Guericke University, Magdeburg, Germany. ERP measures of selective attention, response choice and reaction time were delayed during hypoglycemia compared to baseline performance. After restoration of euglycemia, totalerror frequencies were still higher than at baseline and recovery of the quality of task performance was delayed. Hypoglycemia impairs both the stimulus and the motor-response selection. Large negative shifts in cortical potentials were distributed over frontal areas during hypoglycemia, suggesting that the frontal cortex is involved in the control of attention and stimulus selection. (Smid HGOM, Trumper BG, Pottag G et al. Differentiation of hypoglycaemia induced cognitive impairments. An electrophysiological approach. Brain June 1997;120:1041-1056). (Respond: Dr Smid, Neurophysiology Clinic, Otto-von-Guericke University, Leipzigerstrasse 44, 39120, Magdeburg, Germany).

COMMENT. Hypoglycemia has an adverse effect on attentional behavior by increasing reaction times and error frequencies. After restoration of normal blood sugar levels there is a delay in recovery of quality of task performance. The frontal cortex is more highly activated during acute hypoglycemia as a compensatory mechanism for the reduced energy supply to the brain. Frontal location brain mechanisms, important in control of attention, are particularly affected by the impaired energy supply associated with hypoglycemia. 